\title{
Early Integration of Bilateral Touch in the Primary Somatosensory Cortex
}

\author{
Luigi Tamè, '* Francesco Pavani, ${ }^{1,2}$ Christos Papadelis, ${ }^{3}$ \\ Alessandro Farnè, ${ }^{4,5}$ and Christoph Braun ${ }^{1,2,6,7}$ \\ ${ }^{1}$ Center for Mind/Brain Sciences, University of Trento, Rovereto, Italy \\ ${ }^{2}$ Department of Psychology and Cognitive Sciences, University of Trento, \\ Rovereto, Italy \\ ${ }^{3}$ Department of Newborn Medicine, Children's Hospital Boston, \\ Harvard Medical School, Boston, USA \\ ${ }^{4}$ INSERM U1028, CNRS UMR5292, Bron, France \\ ${ }^{5}$ Université Claude Bernard Lyon I, Lyon, France \\ ${ }^{6}$ MEG-Center, University of Tübingen, Tübingen, Germany \\ ${ }^{7}$ Werner Reichardt Centre for Integrative Neuroscience, University of Tübingen, Tübingen, \\ Germany
}

\begin{abstract}
Animal, as well as behavioural and neuroimaging studies in humans have documented integration of bilateral tactile information at the level of primary somatosensory cortex (SI). However, it is still debated whether integration in SI occurs early or late during tactile processing, and whether it is somatotopically organized. To address both the spatial and temporal aspects of bilateral tactile processing we used magnetoencephalography in a tactile repetition-suppression paradigm. We examined somatosensory evoked-responses produced by probe stimuli preceded by an adaptor, as a function of the relative position of adaptor and probe (probe always at the left index finger; adaptor at the index or middle finger of the left or right hand) and as a function of the delay between adaptor and probe (0, 25, or $125 \mathrm{~ms})$. Percentage of response-amplitude suppression was computed by comparing paired (adaptor + probe) with single stimulations of adaptor and probe. Results show that response suppression varies differentially in SI and SII as a function of both spatial and temporal features of the stimuli. Remarkably, repetition suppression of SI activity emerged early in time, regardless of whether the adaptor stimulus was presented on the same and the opposite body side with respect to the probe.
\end{abstract}

Contract grant sponsors: European Union's Seventh Framework Programme for research [Marie Curie Individual Fellowship, (COFUND) European Commission/Provincia Autonoma di Trento], technological development and demonstration (to L.T.); Contract grant number: 40101908; Contract grant sponsor: ANR11-LABX-0042, ANR-10-IBHU-0003 and FRM-DMP20101120398, and the James S. McDonnell Scholar Award (to A.F.); Contract grant sponsors: Provincia Autonoma di Trento and Fondazione Cassa di Risparmio di Trento e Rovereto.

This research was supported by the Werner Reichardt Centre for Integrative Neuroscience (CIN) at the University of Tübingen. The $\mathrm{CIN}$ is an Excellence Cluster funded by the Deutsche Forschungsgemeinschaft (DFG) within the framework of the Excellence Initia- tive (EXC 307). Furthermore this research was supported as part of the Berstein Focus "Neurotechnology" by the German Ministry for Education and Research, Project BMBF \#01GQ0831, \#16SV5840.

*Correspondence to: Luigi Tamè, Department of Psychological Sciences, Birkbeck, University of London, London WC1E 7HX, United Kingdom. E-Mail: luigi.tame@gmail.com

Received for publication 17 October 2014; Revised 30 November 2014; Accepted 1 December 2014.

DOI: 10.1002/hbm.22719

Published online 16 December 2014 in Wiley Online Library (wileyonlinelibrary.com). 
These novel findings support the notion of an early and somatotopically organized inter-hemispheric integration of tactile information in SI. Hum Brain Mapp 36:1506-1523, 2015 . 2014 Wiley Periodicals, Inc.

Key words: SI; bilateral integration; touch at the fingers; MEG

\section{INTRODUCTION}

Dexterous and well-coordinated bimanual motor tasks require early integration of tactile stimuli from the two sides of the body. This intuitive concept appears to be in contrast with the textbook notion of unilateral representation of tactile input in primary somatosensory cortex (SI) [Nelson and Chen, 2008; Penfield and Boldrey, 1937]. Bilateral integration of tactile information is generally accepted for structures beyond SI, in particular the secondary somatosensory cortex (SII) [Eickhoff et al., 2010]. However, growing evidence in the last decade suggests that also SI contributes to the integration of somatosensory inputs from the two sides of the body [Kanno et al., 2004; Tamè et al., 2012; Tan et al., 2004; Tommerdahl et al., 2006]. Yet, whether SI involvement in the integration of bilateral tactile stimuli occurs early or late during tactile processing remains a matter of debate [Sutherland, 2006].

In animals, bilateral receptive fields have been found in the monkey somatosensory area 2 [Iwamura et al., 2001, 2002], that is considered to be the homologue of Brodmann area 2 of human SI. Moreover, inter-hemispheric interactions have been revealed in SI of owl monkeys, within area $3 b$, for stimuli presented to both paws [Lipton et al., 2006; Reed et al., 2010, 2011]. In rats, SI integrates inputs from the contralateral and ipsilateral whisker pads [Shuler et al., 2001]. In humans, neuroimaging studies using magnetoencephalography (MEG) and functional magnetic resonance imaging (fMRI) have documented neural activity in SI in response to tactile stimulation on the ipsilateral side of the body [Hlushchuk and Hari, 2006; Tan et al., 2004;

\section{Abbreviations}

cSI Contralateral SI

cSII Contralateral SII

ECD Equivalent current dipole

EEG Electroencephalography

fMRI Functional magnetic resonance

GOF Goodness of fit

ISI Inter-stimulus interval

iSI Ipsilateral SI

iSII Ipsilateral SII

ITI Inter-trial interval

MEG Magnetoencephalography

MN Median nerve

RS Repetition suppression

SEF Somatosensory evoked field

SI Primary somatosensory cortex

SII Secondary somatosensory cortex
Tommerdahl et al., 2006]. In a recent study, we examined the contribution of the somatosensory cortices to the spatial coding of touch at the fingers of the same or different hands, taking advantage of fMRI repetition-suppression (RS) paradigm. It is commonly assumed that RS reflects the decrement of neuronal responses that results from the repeated presentation of a stimulus feature to which the neurons are selective [Grill-Spector and Malach, 2001; Krekelberg et al., 2006]. This physiological response was initially described in single cell recordings [Gross et al., 1972; Li et al., 1993; Miller et al., 1991; Tanaka et al., 1991] and has now been largely documented also using fMRI in humans for different modalities such as audition [Belin and Zatorre, 2003; Dehaene-Lambertz et al., 2006], vision [Kourtzi and Kanwisher, 2000; Lingnau et al., 2009a,b; Mahon et al., 2007; Vuilleumier et al., 2002], touch [Li Hegner et al., 2010; Tamè et al., 2012] and cross-modal processes [Chong et al., 2008; Tian and Poeppel, 2013] (for reviews on the fMRI adaptation paradigm see Grill-Spector and Malach, 2001; Grill-Spector et al., 2006; Henson, 2003). In accordance with this principle, in a previous fMRI study we found that stimulating the index finger twice produced more suppression (lower BOLD response) in the somatosensory cortices than stimulating sequentially adjacent fingers (i.e., the middle finger followed by the index finger; [Tamè et al., 2012]. Notably, this effect was present also when pairs of tactile stimuli were delivered to opposite hands, suggesting that SI also can integrate signals originating from the two sides of the body. Despite the prominent contralateral response of SI, this approach has proven very sensitive in revealing the activity in the somatosensory cortices following bilateral tactile stimulation.

The low temporal resolution of fMRI, however, did not allow us to determine whether the integration of contralateral and ipsilateral tactile information occurred in SI at early or late stages of tactile processing. Studies in animals [Iwamura et al., 2001, 2002; Shuler et al., 2001] and more recent works in humans [Borchers et al., 2011; Kanno et al., 2004; Zhu et al., 2007], suggest that touch on the fingers from the two sides of the body might be integrated at an early stage of tactile information processing. Different anatomical pathways as modelled in Figure 1, can mediate this integration in SI [Sutherland, 2006]. A first possibility (direct ipsilateral projection) relies on projections from the receptor surface to ipsilateral SI (iSI), that run in parallel to the ones targeting contralateral SI (cSI) [Kanno et al., 2003, 2004], and that are mediated by uncrossed afferent fibers [Noachtar et al., 1997]. A second possibility (transcallosal projection) is that SI receives ipsilateral somatosensory 


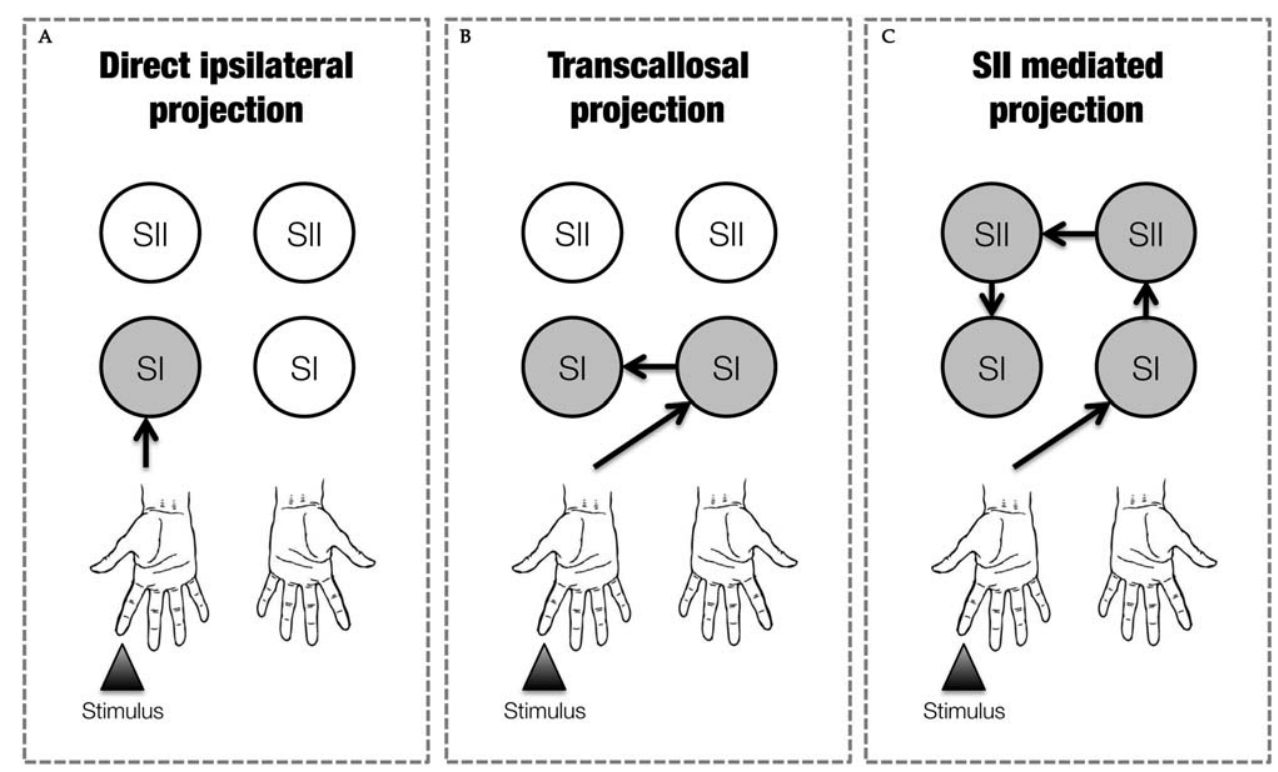

Figure I.

Graphical representation of the possible anatomical pathways mediating the integration processing of tactile information from the two sides of the body. In the example the tactile stimulated finger is the right index. Grey circles represent the passage of information through that particular node. (A) Direct ipsilateral projections, (B) transcallosal projections and (C) SIl mediated projections.

inputs from cSI directly, via transcallosal fibers [Allison et al., 1989; Caminiti et al., 2013; Fabri et al., 2001, 2005; Fling et al., 2013]. Finally, a third possibility (SII mediated projection) is that cortico-cortical modulations of SI could also emerge via transcallosal connections between homotopic SII regions [Schnitzler et al., 1995; Tommerdahl et al., 2006]. These three anatomical pathways-which are clearly not mutually exclusive-are schematically illustrated in Figure 1.

To determine which pathway can better explain the integration of tactile stimuli from the two sides of the body in SI, we investigated the temporal dynamics of the neural response when tactile stimuli were delivered to opposite hands at different delays. To overcome the limited temporal resolution of fMRI, we used MEG in the context of a tactile RS paradigm, similar to the one we used in our previous fMRI study [Tamè et al., 2012]. The rationale for this approach was twofold. First, the RS paradigm already proved very sensitive for revealing neural populations representing the tactile feature of interest [Wühle et al., 2011] which might even not be visible by condition contrasting. Second, the high-temporal resolution (i.e., in the order of milliseconds) of MEG, combined with its good spatial resolution, allows monitoring brain activity immediately after stimulus onset and provides insight in the information retention dynamics within certain brain areas. Specifically, while recording the neuromagnetic activity to a fixed tactile stimulus, we delivered a brief tactile adaptor on the same (i.e., homologous) or different (i.e., non-homologous) finger with respect to the probe. Crucially, the adaptor was delivered on the same hand as the probe (i.e., unilateral stimulation) or on the other hand (i.e., bilateral stimulation). Adaptor and probe stimuli were either presented simultaneously or with a 25 or 125 ms delay.

Predictions were as follows. First, based on the previous literature on SI involvement in the integration of bilateral tactile stimuli reviewed above, we expected attenuation of the neural response both when adaptors and probes were delivered unilaterally and when they were delivered bilaterally. Second, based on behavioural and brain imaging data suggesting that SI contribution to the integration of somatosensory inputs from the two sides of the body occurs in a somatotopically organised fashion [Tamè et al., 2011, 2012, 2013], we expected differential amounts of response suppression as a function of which finger serves as adaptor (i.e., homologous vs. non-homologous), regardless of whether adaptors and probes are unilateral or bilateral. Third, we predicted that RS occurring at different delays would be informative as to which pathway (see Fig. 1) is the main mediator of the between hands interaction. Critical in this prediction is the persistence of the somatosensory signal within SI, which is assumed to last from $60 \mathrm{~ms}$ [Mauguière et al., 1997] to around $110 \mathrm{~ms}$ [Hamada et al., 2002]. If bilateral integration in SI originates from fast pathways (e.g., direct ipsilateral projection or transcallosal transfer from cSI; see Fig. 1A,B), we should expect RS from bilateral stimulation to occur when adaptors and probes are delivered with a short delay $(25 \mathrm{~ms})$. 
Instead, if bilateral integration in SI originates from a slower pathway (transcallosal connection between homotopic SII regions; see Fig. 1C), RS should emerge only when stimuli are delivered with a longer delay (125 ms).

\section{MATERIAL AND METHODS}

\section{Participants}

Twenty right-handed participants (mean age $=27$; $\mathrm{SD}=4$; six females) participated in the study. Handedness was inferred from participants' self-reports. All participants reported normal or corrected to normal vision. Furthermore, they reported no history of somatosensory impairment, psychiatric or neurological disorders, and no current use of any psychoactive medications. Participants gave their informed written consent before participating in the study that was carried out according to 1964 Declaration of Helsinki (last update: Seoul, 2008). The study was approved by the ethical committee of the University of Trento. Three participants were discarded from the analysis because of a too low activity in the probe-only conditions.

\section{Stimulation}

To study somatosensory evoked magnetic brain activity, participants were stimulated by short tactile pulses of skin indentation applied to the finger tips. One or two fingers were stimulated at a time or in sequence. Tactile stimuli were provided by a four channels piezo-electric stimulator (Quaerosys, Schotten, Germany, www.quaerosys.de) that was placed outside the shielded room hosting the MEG system. Tactile stimuli were set clearly above threshold to specifically avoid extinction-like effects, reported so far only under weak tactile stimulation [Farnè et al., 2007; Jacobs et al., 2011]. In general, participants reported to perceive the double tactile stimulations in all conditions. The code for the stimulation sequence was downloaded from the control computer to the stimulator before the beginning of a trial. The output of the stimulation sequence was initiated by a trigger signal. The digitization rate of the output signal was $2 \mathrm{kHz}$. To avoid introduction of noise into the shielded room, the cables used to connect the stimulator outside the shielded room with the stimulator modules attached to the subject's hand were twisted and a series of ferrite low-pass filters was applied. The four stimulation modules in contact with the participant's finger consisted of a matrix of $2 \times 5$ rods ( $1 \mathrm{~mm}$ in diameter), poking from a flat surface of $4 \times 8 \mathrm{~mm}^{2}$. The six central rods of the matrix protruded for 12 (ms), producing a clearly perceivable skin indentation. The intensity of the tactile pulse was always set to the maximum level available, except for $8 \%$ of the trials that served as catch trials for the behavioural task. Stimuli in catch trials were delivered at half intensity. The stimulation modules were attached to the finger pads of the middle and index finger of either hand using Velcro tape, to ensure constant contact between the fingers and the stimulation modules throughout the experiment. Stimulation modules were placed on a woollen pad to attenuate any possible acoustic noise and to avoid any possible unwanted mechanical transfer of the vibrations between hands and fingers. The hands were positioned in a comfortable posture on the participants' legs. The index and middle fingers of each hand rested on the stimulation modules fixed with the Velcro type.

During the experimental session, instructions were presented by means of a video projector (Panasonic PTD7700E). The projector was placed outside the shielded room and projected the verbal instructions onto a pellucid screen placed in front of the participants $(1.2 \mathrm{~m})$. Instructions were presented at the beginning of the experiment. To avoid eye movements, subjects were asked to gaze at a fixation cross (a green cross of $4.3^{\circ}$ of visual angle) that was presented during each run (see procedure for a complete description of the visual conditions) in the middle of the pellucid screen. The participants' right thumb was placed on an optical response device measuring the changes of the reflected infrared light that were induced by lifting the thumb. The response signal was recorded directly by the MEG acquisition system. While stimulus presentation was controlled by a stimulation computer, the response collection was achieved by the recording software of the MEG (ELEKTA Neuromag).

Throughout the experiment, white noise was presented binaurally using non-magnetic custom earphones connected to a computer (Dell T3400; Core Duo 2 Quad). The acoustic noise signal was used to mask any sounds produced by the operation of the tactile stimulation modules. The computer generating the noise was placed outside the shielded room.

\section{Procedure}

Participants sat in a sound-attenuating, magnetically shielded room. Visual and tactile stimulations were programmed using the in-house software "ASF" [Schwarzbach, 2011], based on the MATLAB Psychtoolbox-3 [Brainard, 1997] for Windows. The experiment comprised 10 separate runs consisting of 240 trials each. In each run, the 15 experimental conditions were repeated 15 times resulting in a total of 225 trials per run. Participants' attention towards the probe stimulus was checked in additional 15 trials per run, yet excluded from further analyses. At the beginning of each trial a green fixation cross appeared at the middle of the pellucid screen, and remained visible for the entire duration of the trial. $500 \mathrm{~ms}$ after the appearance of the fixation cross, depending on the experimental condition, one or two consecutive or simultaneous tactile stimulations were delivered to the participants' fingers, each lasting $12 \mathrm{~ms}$ (A, adaptor stimulus; P, probe stimulus, 


\section{Stimulation Trials}

A Adaptor stimulus $\quad \mathrm{P}$ Probe stimulus

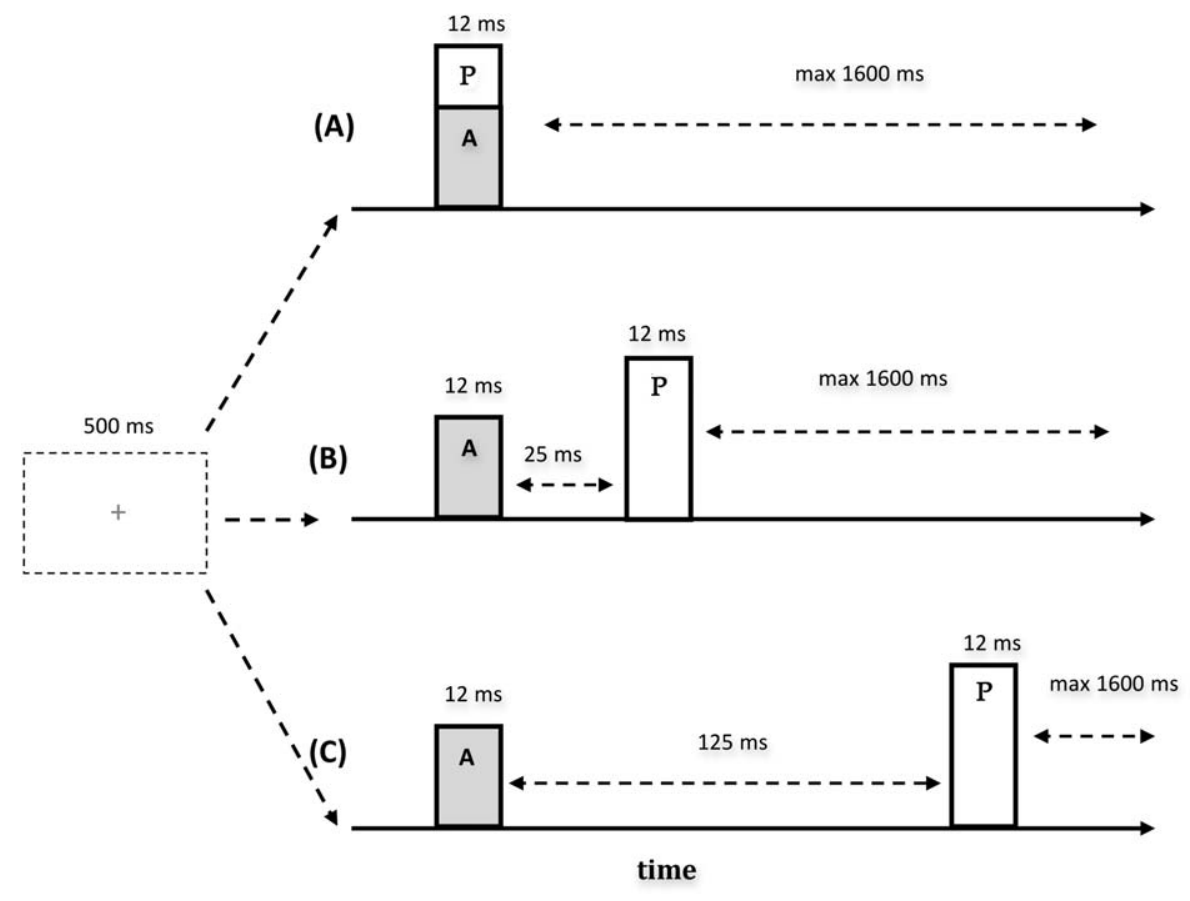

Figure 2.

Scheme of stimulation sequences. Pairs of short tactile stimuli (12 ms) were presented at different ISIs (A) simultaneously (B) with an ISI of $25 \mathrm{~ms}$ or (C) with an ISI of $125 \mathrm{~ms}$. The start of the stimulation phase was indicated by a green fixation cross in the middle of the screen $500 \mathrm{~ms}$ before the adaptor stimulus.

see Fig. 2). A and $P$ occurred at either the same time, or separated by an inter-stimulus interval (ISI) of $25 \mathrm{~ms}$ (short ISI) or $125 \mathrm{~ms}$ (long ISI). $T$ was followed by an inter-trial interval (ITI) ranging randomly from $400 \mathrm{~ms}$ to $2000 \mathrm{~ms}$. Note that the second stimulus (probe) was always applied to the left index finger. The fifteen experimental conditions fell in four main classes: (1) sequential presentation of the adaptor and probe stimulus at the short interval; (2) sequential presentation at the long interval; (3) simultaneous presentation and (4) single finger stimulation. The first two classes included four different stimulation conditions each as a function of whether the stimulation was repeated on the same or different fingers; and whether $\mathrm{A}$ and $\mathrm{P}$ were delivered to the same (unilateral stimulation) or the opposite hands (bilateral stimulation) (see Table I): (a) A and P were delivered on the same finger of the same hand (unilateral stimulation) (i.e., left index finger, condition short/long Li-Li; Fig. 3a); (b) A and $\mathrm{P}$ were delivered on non-homologous fingers of the same hand (unilateral stimulation) (i.e., left middle and left index fingers, condition short/long Lm-Li; Fig. 3b); (c) $\mathrm{A}$ and $\mathrm{P}$ were delivered on the homologous fingers of opposite hands (bilateral stimulation) (i.e., right index and left index fingers, condition short/long Ri-Li; Fig. 3c); (d) $\mathrm{A}$ and $\mathrm{P}$ were delivered on non-homologous fingers of opposite hands (bilateral stimulation) (i.e., right middle and left index fingers, condition short/long Rm-Li; Fig. 3d). The third class (i.e., simultaneous stimulation) involved only three of the previously described stimulation sequences. Simultaneous presentation of adaptor and probe stimulus (i.e., Li-Li) could not be realized and was therefore not included in this class. Finally, the fourth class (i.e., singles stimulation) included four conditions in which individual fingers where stimulated alone (see Table I): stimulation of the left index finger alone (condition Li; Fig. 3g); stimulation of the left middle finger alone (condition Lm; Fig. 3e); stimulation of the right index finger alone (condition Ri; Fig. 3h); stimulation of the right 
- Early Integration of Bilateral Touch in SI •

TABLE I. Stimulation conditions

\begin{tabular}{llll}
\hline & \multicolumn{1}{c}{$(1)$} & \multicolumn{1}{c}{$(2)$} & $(3)$ \\
Condition & $\begin{array}{l}\text { Short inter-stimulus } \\
\text { interval }(25 \mathrm{~ms})\end{array}$ & $\begin{array}{l}\text { Long inter-stimulus } \\
\text { interval }(125 \mathrm{~ms})\end{array}$ & Simultaneous \\
\hline (a) & short Li-Li & long Li-Li & - \\
(b) & short Lm-Li & long Lm-Li & $\mathrm{Lm}-\mathrm{Li}$ \\
(c) & short Ri-Li & long Ri-Li & $\mathrm{Ri}-\mathrm{Li}$ \\
(d) & short Rm-Li & long Rm-Li & $\mathrm{Lm}$ \\
\hline
\end{tabular}

middle finger alone (condition Rm; Fig. 3f). The fifteen experimental conditions were presented in a pseudorandom order. It was assured that the probability for each experimental condition to be preceded by the same condition or any different condition was fifty percent [Kourtzi and Kanwisher, 2000; Tamè et al., 2012]. This procedure ensured that each pair of stimuli forming the different stimulation conditions had the same weight, avoiding that results in the response were affected by the fact that one condition occurred in a certain position more often than another.

To control for participants' attention, a written question appeared on the screen ("Did you feel the weak stimulation on your left index finger?") in fifteen catch trials per run, for a total of 150 trials in the whole experiment (on a total of 2,550 trials). These catch trials were excluded from the analysis.

\section{MEG Recording}

Magnetic brain responses were recorded continuously for 320 s per run with the 306-channels (204 first order planar gradiometers, 102 magnetometers) whole-head MEG system (Elekta Neuromag Vectorview 306) in a magnetic shielded room (AK3B, Vakuum Schmelze, Hanau, Germany). The continuous recording was digitized at $1 \mathrm{kHz}$ using a low-pass filter set at $330 \mathrm{~Hz}$.

\section{Analysis}

\section{Preprocessing}

The continuous recording was filtered off-line with a 40 $\mathrm{Hz}$ low-pass filter and a $1 \mathrm{~Hz}$ high-pass filter and segmented into trials of $650 \mathrm{~ms}$. Each segment included the neuromagnetic responses evoked by the tactile stimulations and a pre-stimulus baseline of $100 \mathrm{~ms}$ that was set before the first tactile stimulus of each trial. Somatosensory evoked fields (SEFs) were time-locked to the presentation of the first stimulus and averaged across trials and recording blocks, separately for all stimulation conditions (see Fig. 3). The activity of the baseline defined as the time interval ranging from 0 to $50 \mathrm{~ms}$ before the onset of the first stimulus was subtracted from the whole trial. Participants with an activity in the probe alone stimulation condition lower than $4.5 \mathrm{nAm}$ were excluded from the analysis. Using this criterion, three participants were discarded.

\section{Estimation of sources}

Data analysis focused on the relative changes in neuromagnetic responses of planar gradiometer sensors evoked by the second stimulus, the probe, which was always the left index finger and which was either presented alone or together with an adaptor stimulus (1) simultaneously, (2) $25 \mathrm{~ms}$, or (3) $125 \mathrm{~ms}$ before the probe stimulus, respectively. To capture amplitude changes in SI and SII ipsiand contralateral to the presentation of the probe stimulus an equivalent current dipole (ECD) approach was used. To compare source activities across all conditions, a four dipoles model (SI and SII on both hemispheres) was fitted to the averaged evoked fields of the four single stimulation conditions, the index and middle finger stimulation of both hands. Since source location and source activity are strongly related we refrained from estimating individual dipole source solutions for each of the single stimulus conditions. Variations in source localization for different stimulation conditions might confound the estimated source activity and thus corrupt the comparison of the source activities across different stimulation conditions. To have an adequate signal-to-noise ratio, the source estimation was based on a total of 600 trials (150 per each of the single stimulation condition) (see Fig. 4a).

Two clear distinct components were observed in the SEF [Hari et al., 1984], one peaking at the latency range between 20 and $60 \mathrm{~ms}$ (mean peak: $35 \mathrm{~ms}$ ) and another from 60 and $90 \mathrm{~ms}$ (mean peak: $70 \mathrm{~ms}$ ) in both hemispheres. Analysis of the peaks latency in SII revealed that response was $6.7 \mathrm{~ms}$ faster in the contralateral compared with the ipsilateral hemisphere $[t(16)=-3.31, P=0.004)$. This delay is compatible with the somatosensory transfer time across the corpus callosum [Aboitiz et al., 1992; Caminiti et al., 2013; Stancak et al., 2002] and corroborate the suitability of our approach. ECDs were fitted at the peak of each component for each participant by using the BESA software (BESA Research 5.3). Representations of index and middle fingers of one hand were not distinguished and were modeled as a common dipolar source. In this way, we avoided incorrect assignments of activity to the index and the middle finger to the two dipoles. Modelling 


\section{Double Stimulations}



Adaptor stimulus

Adaptor stimulus

B

\section{$\underline{\text { Single Fingers stimulation }}$}


\section{Probe stimulus}

Figure 3.

Schematic representation of paired (A) and single (B) stimulation conditions. The empty circle represents the test stimulus, while the colored circles represent the adaptor stimuli. (a) Stimulation condition in which the left index was stimulated twice $(\mathrm{Li}+\mathrm{Li}$; within hand stimulation); (b) Stimulation condition in which the left index and middle fingers were stimulated $(\mathrm{Lm}+\mathrm{Li}$; within hand stimulation); (c) Stimulation condition in which the two index fingers were stimulated $(\mathrm{Ri}+\mathrm{Li}$; between hand stimulation); (d) Stimulation condition in which the right middle and left index fingers were stimulated $(\mathrm{Rm}+\mathrm{Li}$; between hand stimulation); (e) Stimulation condition in which the left middle finger was stimulated alone; ( $f$ ) Stimulation condition in which the right middle finger was stimulated alone; (g) Stimulation condition in which the left index finger was stimulated alone; (h) Stimulation condition in which the right index finger was stimulated alone. the representation of both fingers with a common source will introduce only minor fitting errors as shown by the goodness of fit (GOF) values which are for all participants and conditions better than $88 \%$ in SI and $82 \%$ in SII. Moreover, using one dipole for both finger representations may introduce an error in the source activity estimate. However, this error will be systematic, and will affect all conditions equally.

For dipole fitting, we used a spherical headmodel that was derived from participants' headshapes and that was determined before the MEG recording by using a Polhemus 3d-digitization system Fastrak digitizer (Polhemus, Colchester, VT). The anatomical correspondence of the dipole locations (Fig. 4b) was identified by using the cytoarchitectonic maps following a methodology we already used successfully [Papadelis et al., 2011]. Figure 4c indicates the correspondence of ECDs with the cytoarchitectonic maps of BA3b $(80 \%$ for right SI and $70 \%$ for left SI) and OP1 (70\% for right SII and 70\% for left SII) for one participant. Moreover, the SEFs for unilateral and bilateral stimulation conditions in the different delays and their correspondent topographic maps of a participant are shown in Figure 5.

\section{Estimation of virtual channels}

To estimate the activity of the dipole sources, which are referred to as virtual channels cSI, iSI, contralateral SII (cSII) and ipsilateral SII (iSII), the leadfields (i.e., the matrices describing the relationship between the activity of a single dipole of unitary strength and the sensor level activation) of the four dipoles were calculated from the positions and orientation of an ECD and the head model for each participant. The relation between the magnetic field and the source activity is given by the following equation: $B=L S+\varepsilon$, with $B$ being an $m \times n$ matrix describing the measured time course of magnetic activity ( $n$ samples) at $m$ sensors. $S$ is a $k \times n$ matrix containing the time course of the $k$ dipolar sources. $L$ is an $m \times k$ lead field matrix and $\varepsilon$ reflects the noise activity which is assumed to be Gaussian as first approximation. Using linear regression analysis, activity of the virtual channel was estimated: $S=\left(L^{\prime} L\right)^{-1}$ $L^{\prime} B$, where $(.)^{\prime}$ denotes the transpose and $(.)^{-1}$ the inverse of a matrix. In total there were 1,020 waveforms corresponding to four sources, 15 conditions and 17 participants.

To assess the effect of the adaptor stimulus on the processing of the probe stimulus, we subtracted the source activity of the adaptor (i.e., activity derived from single finger stimulation) from the compound source activities evoked by the paired finger stimulation (adaptor + probe) for all double stimulation conditions. The probe stimulus evoked source activity for each stimulation condition was again baseline corrected by subtracting the mean activity of the $50 \mathrm{~ms}$ period before probe stimulus onset.

For statistical analyses, peak amplitudes and latencies of the ECDs' source activities were defined. For right SI, which is contralateral to the application of the probe 

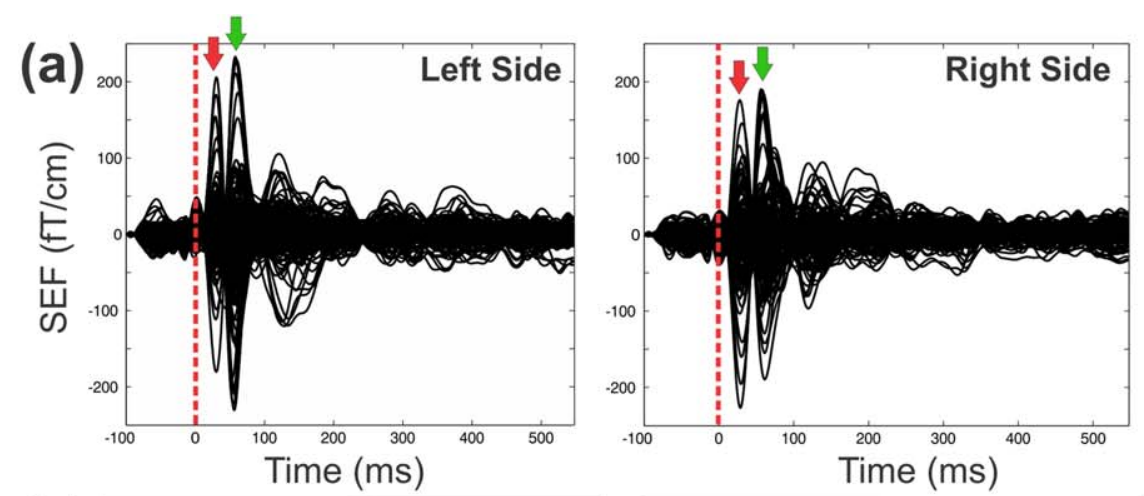

(b)
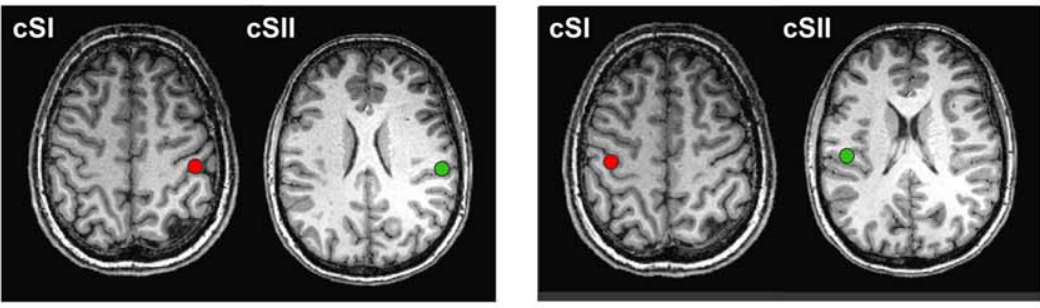

(c)


(d)
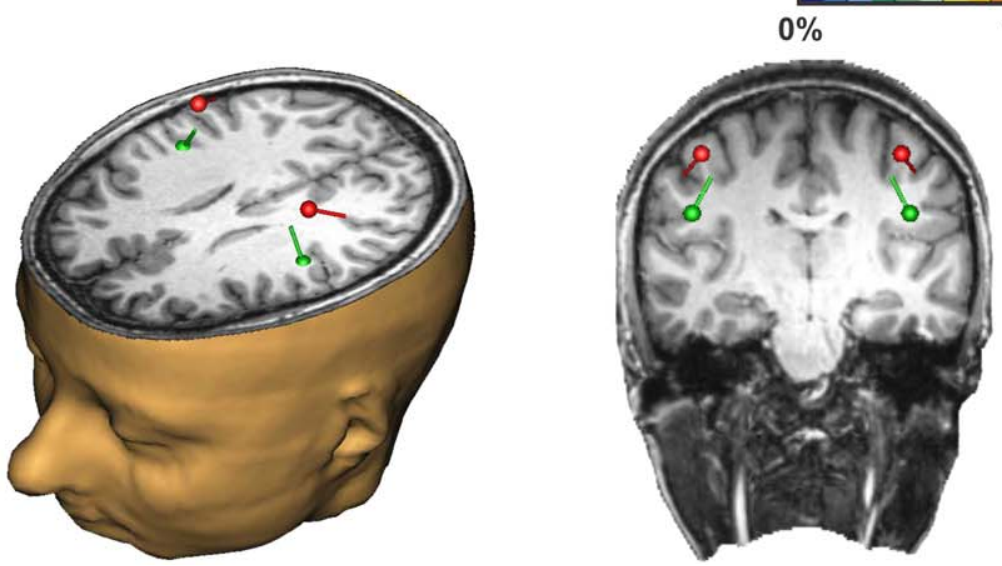

Figure 4.

Schematic representation of the ECD approach used to define the four ROls in the contra- and ipsi-lateral SI and S2. (a) SEFs for the single finger stimulation (150 trials) of the left (left panel) and right (right panel) index and middle fingers respectively. Red and green arrows indicate the latencies at which the ECDs were estimated. (b) The corresponding ECDs located at SI and SIl for the left (left panel) and right (right panel) stimulation sides fitted at the peak of the first and second SEF components. MRI slides are in neurological orientation. (c) The correspondence of the two ECDs with the underlying cytoachitectonic maps (Eickoff et al., 2006). Red and green ECDs are overlaid on BA3b and OPI maps respectively. (d) 3D representation of the four ECDs locations with respect to the participant's anatomical scan. 

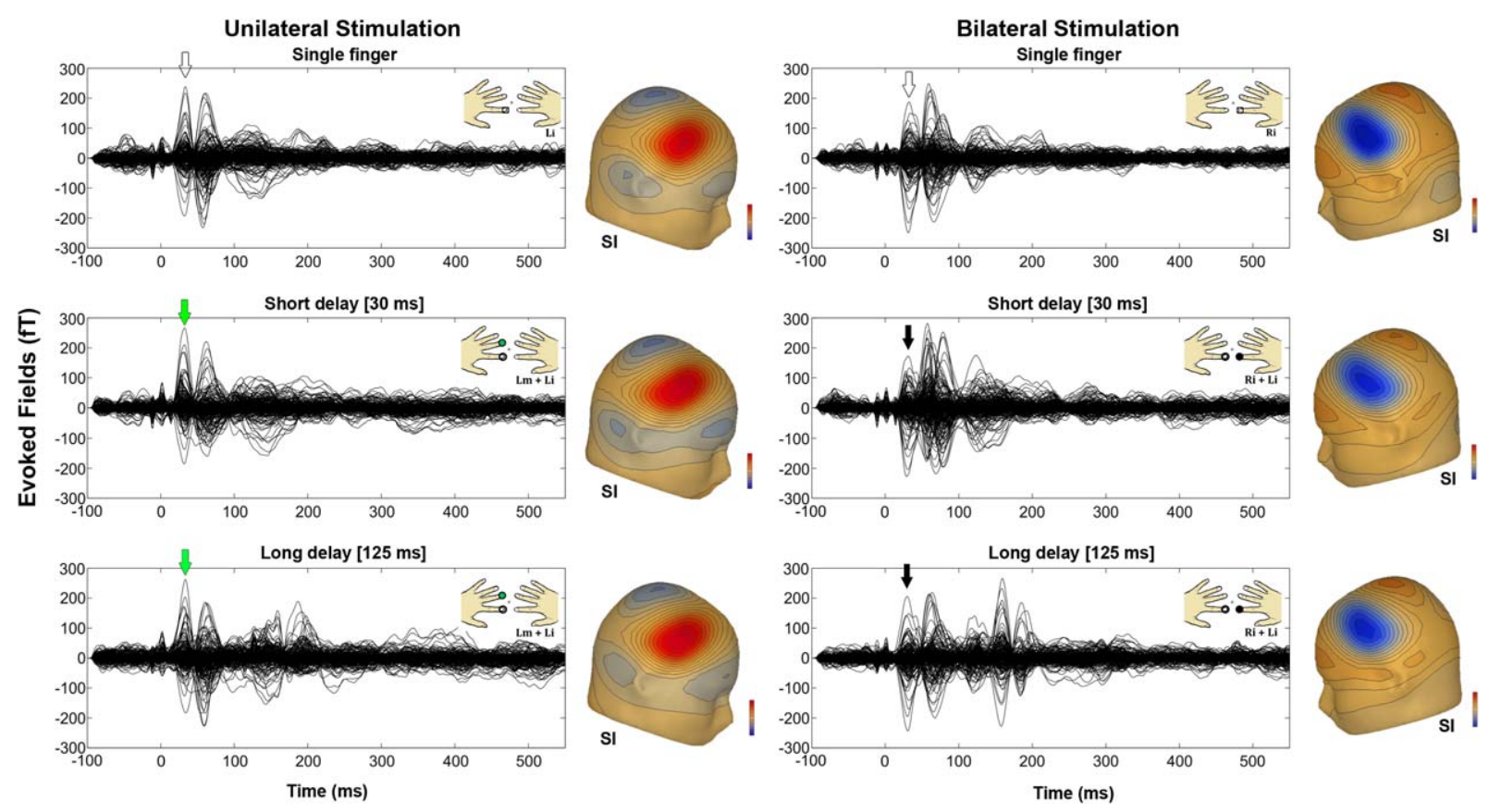

Figure 5.

SEFs for the unilateral (left panels) and bilateral (right panels) stimulation of the single and double short $(30 \mathrm{~ms})$ and long $(125 \mathrm{~ms})$ fingers stimulation. The corresponding topographic maps of the SEFs in the SI are also shown aside. Green and black arrows indicate the latencies at which the maps were depicted.

stimulus peaks were determined in a window between 20 and $60 \mathrm{~ms}$; for cSII the window ranged from 47 to $85 \mathrm{~ms}$ and for iSII from 50 to $90 \mathrm{~ms}$; since for iSI activation no peaks were obvious and thus no peak parameters were determined.

\section{Statistics}

To quantify the amount of RS, dipole activity peak values for each DS conditions $P_{\mathrm{x}}$ were standardized by dividing them by the peak values obtained for the left index finger only condition $P_{\text {li }}$ (i.e., response to the probe). Data were presented as percentage of suppression from index finger only trial (see Fig. 6). This normalisation procedure accounted for the variability of the signal strength across participants.

$$
r_{x}=\frac{P_{l i}-P_{x}}{P_{l i}} * 100
$$

To examine whether the dipole activity (i.e., activity of cSI, cSII, and iSII) was modulated as a function of the tactile DS conditions (different fingers and timing stimulation) we subjected the strength of response suppression for the different adaptor conditions ( $\mathrm{Lm}-\mathrm{Li}, \mathrm{Li}-\mathrm{Li}, \mathrm{Rm}-\mathrm{Li}$, and $\mathrm{Ri}-\mathrm{Li}$ ) to separate repeated measures ANOVAs. For each dipole activity (cSI, cSII, and iSII) two ANOVAs where computed, one for the within hand and one for the between hands stimulation. The "within hand" ANOVAs used Delay (short, long) and FingER (homologous, non-homologous) as within factors, whereas the "between hands" ANOVAs applied Delay (simultaneous, short, long) and FINGER (homologous, nonhomologous) as within participant factors. To test whether tactile DS conditions produced suppression we further performed a series of one-tailed $t$-tests comparing the dipole activity resulting from the probe minus the DS conditions (i.e., probe-adaptor) for each of the sources (cSI, cSII, and iSII) against the activity in the condition in which the probe stimulus was presented alone (i.e., probe only). Tukey HSD test was used for all post-hoc comparisons.

\section{RESULTS}

Using a dipole source model we characterised the wellknown stimulus-specific activation represented by activity in the cSI, cSII, and iSII (see Fig. 4), as reported by previous studies [Wühle et al., 2011]. During the subsequent steps of the analysis, the RS of the probe stimulus was determined as a function of which adaptor stimuli preceded the probe. Figures 5 and 6 shows the response suppression (\% reduction in amplitude) for the different sources and for the different timings of the adaptor with respect to the probe stimulus (simultaneous, short: $25 \mathrm{~ms}$, and long: $125 \mathrm{~ms}$ ).

\section{Primary Somatosensory Cortex}

While cSI, with respect to the probe stimulus, revealed a clear response, no evoked signal was found for iSI. 

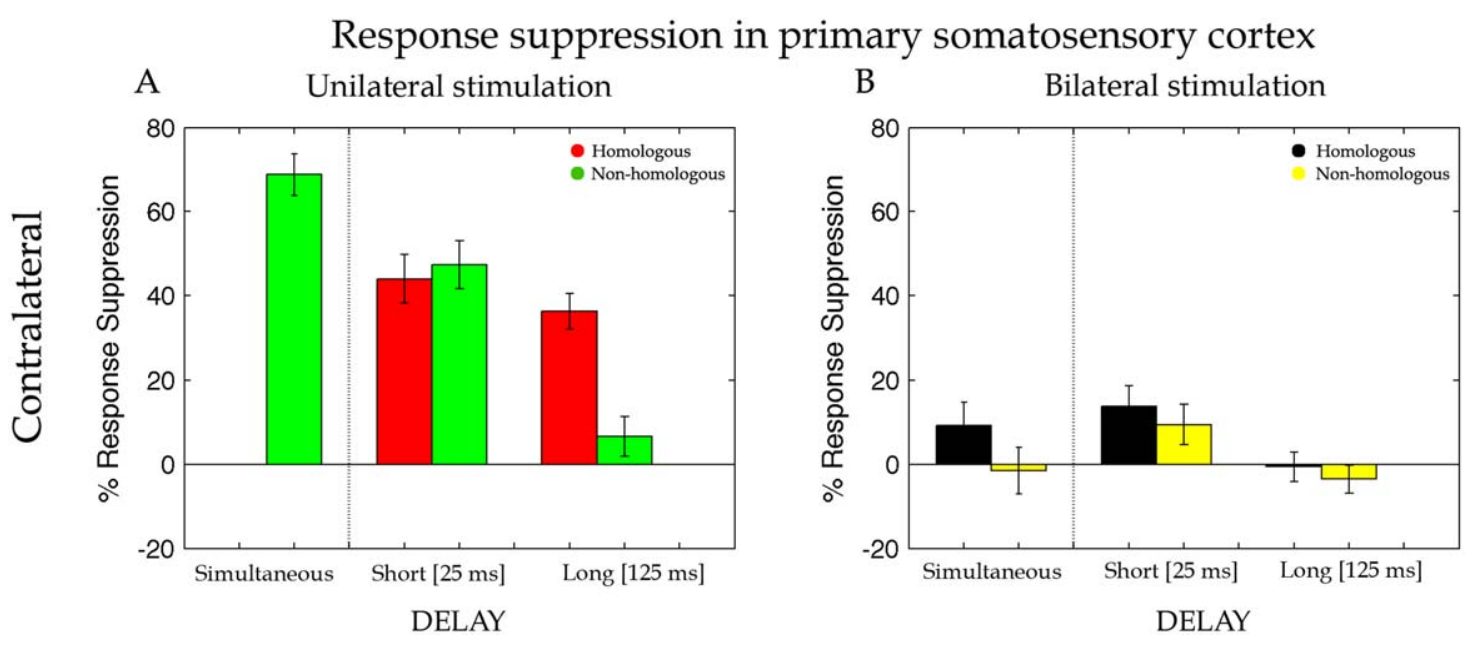

Figure 6.

Percentage of response suppression of the dipole activity in the cSI when the test stimulus was preceded by an adapting stimulation on the homologous ( $\mathrm{Li}-\mathrm{Li}$, red bars) and non-homologous (Lm-Li, green bars) finger of the same hand and on the homologous (Ri-Li, black bars) and non-homologous (Rm-Li, yellow

Therefore, virtual channel iSI was discarded from further analyses. To assess the validity of our approach, we started by examining RS when both stimuli were delivered within the same hand (i.e., unilateral stimulation). On the basis of previous reports on animals [Simons et al., 2007] and our own fMRI study [Tamè et al., 2012], we expected a change in RS recorded in the SI contralateral to the presentation of the probe stimulus side (cSI) when the adaptor was applied to the same finger as the probe, compared with when the adaptor was applied to the neighbouring finger [Tamè et al., 2012; Wühle et al., 2010, 2011]. To test this hypothesis, RS values evoked by the probe stimulus in cSI for unilateral hand stimulation were entered into a repeated measure ANOVA with DeLAy (short, long) and FINGER (homologous, non-homologous) as within participant factors. Note that for this analysis the factor Delay did not include the simultaneous condition because it cannot be implemented with unilateral stimulation (see "Materials and Methods" section). Stronger RS emerged for homologous $(M \pm S E=40 \% \pm 5)$ compared with nonhomologous $(M=27 \% \pm 6)$ fingers stimulation (main effect of FingER, $F(1,16)=8.42, \quad P=0.01, \quad \mathrm{MSE}=0.04$, $\left.\eta_{\mathrm{p}}{ }^{2}=0.35\right)$. Moreover, a significant interaction between Delay and Finger emerged $[F(1,16)=23.11, P=0.0002$, MSE $\left.=0.02, \eta_{\mathrm{p}}{ }^{2}=0.60\right]$. For the short-delay $(25 \mathrm{~ms})$, RS was similar for homologous $(M=44 \% \pm 6)$ and nonhomologous fingers $(M=47 \% \pm 6, P=0.9)$. By contrast, for the long-delay (125 ms) RS persisted for the homologous finger $(M=36 \% \pm 4)$, but was drastically reduced for the non-homologous finger stimulation $(M=7 \% \pm 5)$. Instead, in the long-delay, suppression lasted longer for the repeated stimulation of the same finger than for adap- bars) finger of the opposite hand simultaneously (Sim), with a short interval of $25 \mathrm{~ms}$ (Short) and with a long interval of 125 ms (Long). Error bars represent the standard errors of the mean $( \pm$ SEM).

tor stimulation applied to the other finger. A main effect of Delay, $F(1,16)=19.27, \quad P=0.0005, \quad \mathrm{MSE}=0.05$, $\eta_{\mathrm{p}}{ }^{2}=0.55$, was also present, but subsidiary to the higher order interaction described above. For completeness we also compared the response evoked by simultaneous presentation of probe and adaptor to adjacent fingers (i.e., adaptor on the left middle finger, probe on the left index finger) with the stimulation of the probe finger alone. A paired $t$-test showed a significant RS effect also in this simultaneous stimulation condition $(M=69 \% \pm 5$; $\left.t_{(16)}=-13.83, P=0.0001\right)$.

This preliminary analysis revealed that (1) RS emerge reliably in CSI when adaptor and probe are delivered to the same hand and (2) RS is modulated as a function of whether the adaptor is applied to the same finger as the probe (homologous stimulation) compared with the neighbouring finger (non-homologous stimulation). Next, we tested whether comparable profiles of RS modulations emerged in cSI also when stimuli were applied to opposite hands. ${ }^{1}$ To this aim, RS values evoked in cSI for bilateral hand stimulation (i.e., adaptor stimulus on the right hand and probe stimulus on the left hand) were entered into a repeated measure ANOVA with DeLAY (simultaneous,

${ }^{1}$ Separate analyses for the unilateral and bilateral stimulation of the two fingers were also motivated by the fact that they elicit a substantially different level of activation and therefore response suppression $(M=29 \% \pm 1)$ in the two hemispheres due to the presence of primarily contralateral projection. This difference can be appreciated by visually comparing the left and right panels of Figure 6A. A $t$-test of cSI activity suppression between unilateral and bilateral stimulation conditions confirms this difference $\left(t_{(16)}=8.89, P=0.0001\right)$. 



Figure 7.

Percentage of response suppression of the dipole activity in the cSIl and iSIl when the test stimulus was preceded by an adapting stimulation on the homologous ( $\mathrm{Li}-\mathrm{Li}$, red bars) and nonhomologous ( $\mathrm{Lm}-\mathrm{Li}$, green bars) finger of the same hand and on the homologous (Ri-Li, black bars) and non-homologous (Rm-Li,

short, long) and FINGER (homologous, non-homologous) as within participant factors. When adaptor and probe stimulus were on opposite hands, results showed a fingerspecific RS caused by significantly greater suppression in the dipole activity for homologous $(M=8 \% \pm 6)$ compared with non-homologous $(M=1 \% \pm 6)$ fingers stimulation (main effect of FINGER, $F(1,16)=9.79, P=0.006, \mathrm{MSE}=0.01$, $\eta_{\mathrm{p}}{ }^{2}=0.38$; see Fig. 6). This indicates that finger homology between adaptor and probe matters also when the two stimuli were delivered to opposite sides of the body, at early as well as later stages of tactile processing. Moreover, RS was modulated by the temporal interval between adaptor and probe [main effect of DELAY, $F(2,32)=7.54$, $P=0.002$, MSE $\left.=0.02, \eta_{\mathrm{p}}{ }^{2}=0.32\right]$. The suppression associated with the short-delay $(M=12 \% \pm 4)$ was stronger than the one associated with the long-delay $(M=0 \% \pm 3$; $P=0.001)$ and tended to be stronger than the one associ-

yellow bars) finger of the opposite hand simultaneously (Sim), with a short interval of $25 \mathrm{~ms}$ (Short) and with a long interval of $125 \mathrm{~ms}$ (Long). Error bars represent the standard errors of the mean $( \pm S E M)$.

ated with the simultaneous stimulation $(M=4 \% \pm 4$; $P=0.08)$. Although it might seem from the bar graph depicted in Figure 6B that some degree of suppression might be present under simultaneous bilateral homologous fingers stimulation, this did not turn out to be statistically significant $(P=0.11)$. No other significant effects or interactions were found $(P>0.5)$. These results indicate that despite the mainly contralateral representation of distal tactile information in SI there seems to be some early interaction between hemispheres. The different suppression in the short and long-delay indicates the presence of interactions at the very early stages of the cortical information process of tactile stimuli (i.e., $25 \mathrm{~ms}$ ). The differential effect as a function of stimulation of homologous vs. non-homologous fingers indicates that this interaction reflects some features of somatotopic organization. 


\section{Secondary Somatosensory Cortex (Contralateral to the Side of the Probe Stimulus)}

Figure 7 shows that the suppression of the dipole activities for unilateral and bilateral stimulation of the hands in SII. Visual comparison of the left and right panels clearly shows a similar profile for ipsilateral and CSII, unlike our findings in SI. This was confirmed by a $t$-test (see Fig. 7B; $\left.t_{(16)}=-1.13, P=0.9\right)$ indicating that strength of activity suppression was comparable for the two hemispheres at the level of SII.

The suppression of the dipole activity in SII under unilateral stimulation was entered into a repeated measure ANOVA with Delay (short, long) and FINGER (homologous, non-homologous) as within participant factors. Differently from cSI, the analysis showed weaker RS of the dipole activity for the homologous $(M=27 \% \pm 16)$ compared with the non-homologous $(M=56 \% \pm 11)$ fingers stimulation (main effect of FingER, $F(1,16)=5.84, \quad P=0.03, \quad \mathrm{MSE}=0.25$, $\left.\eta_{\mathrm{p}}{ }^{2}=0.27\right)$. Evidently, the finger dissociation between shortand long-delay that was found for cSI (see above) vanished in SII and was replaced by a general reduction in the strength of the suppression. No other significant main effects or interactions were found (all ps $>0.2$ ). As in SI, the simultaneous stimulation of the index and middle finger at the same hand showed a strong suppression effect $\left(M=55 \% \pm 12 ; t_{(16)}=-4.68, P=0.0001\right)$ compared with the probe-only stimulation on paired $t$-test.

Effects of bilateral stimulation on RS measured in cSII were, as for SI, analysed by including three levels for the factor Delay (simultaneous, short and long) and Finger (homologous, non-homologous) as within participant factors of a repeated measure ANOVA. This analysis revealed a main effect of DELAY $[F(2,32)=9.40, P=0.0006$, MSE $\left.=0.15, \eta_{\mathrm{p}}{ }^{2}=0.37\right)$ caused by a stronger suppression of the dipole activity for the short- $(M=47 \% \pm 12)$ and long- $(M=39 \% \pm 18)$ compared with the simultaneous $(M=8 \% \pm 12$; $p s=0.007)$ delay. With respect to cSI, here there was an increase of the suppression for the longdelay that indicates a longer persistence of the effects of bimanual stimulation in the cSII than for cSI. No others main effects or interactions resulted to be statistically significant.

\section{Secondary Somatosensory Cortex (Ipsilateral to the Side of the Probe Stimulus)}

For SII ipsilateral to the probe stimulus a $t$-test revealed more suppression for the bilateral compared with unilateral stimulation conditions $\left[t_{(16)}=-2.53, P=0.022\right.$; see Fig. 7D). As for cSII source (Fig. 7A), also for iSII RS values for the unilateral stimulation were entered in a repeated measure ANOVA with Delay (short, long) and FINGER (homologous, non-homologous) as within participant factors. Unilateral stimulation produced weaker suppression for the homologous $(M=19 \% \pm 23)$ compared with the non-homologous
$(M=53 \% \pm 9)$ fingers (main effect of FINGER, $F(1,16)=6.74$, $\left.P=0.02, \mathrm{MSE}=0.31, \eta_{\mathrm{p}}{ }^{2}=0.30\right)$. No others main effects or interactions resulted to be statistically significant. A main effect of DeLAY was nearly significant $F(1,16)=3.77, P=0.07$, MSE $=0.17, \eta_{\mathrm{p}}{ }^{2}=0.19$, showing a tendency toward a weaker suppression for the short- $(M=26 \% \pm 19)$ compared with the long-delay $(M=46 \% \pm 12)$. Instead, analysis on the bilateral stimulation (Fig. 5B) revealed neither statistically significant main effects nor interaction (all $p s>0.2$ ). Compatible to the results for cSI and cSII, the simultaneous stimulation of the probe and its neighbour finger showed a strong suppression effect $(M=40 \% \pm 6 ; P=0.0001)$ compared with probe-only stimulation.

\section{DISCUSSION}

We used a MEG based RS approach to clarify whether touches delivered at both hands are integrated already in SI and whether this integration occurs according to "somatotopic" rules. We found reduction of the neuromagnetic activity in contralateral primary and bilateral secondary somatosensory cortices (i.e., cSI, cSII, and iSII) when the adaptor preceded the probe, compared with when the probe was presented alone. The within hand RS is consistent with previous works [Grill-Spector and Malach, 2001; Otsuru et al., 2011; Tamè et al., 2012; Tanaka et al., 1991] showing a decrement of the neuronal response after repeated presentation of a stimulus feature to which neurons are selective. Importantly, also a between hands RS emerged when stimuli were presented on different sides of the body. Furthermore, when the adaptor and probe were on different hands RS was somatotopically constrained, as it was larger for stimulation of homologous as compared with non-homologous fingers.

We also took advantage of the high-temporal resolution of MEG to investigate the timing of the between hands interactions. The second novel finding reported here is that RS occurs in SI at short delays between adaptor and probe. RS evoked by bilateral stimulation emerged when adaptor and probe were separated by $25 \mathrm{~ms}$, but not when they were separated by $125 \mathrm{~ms}$ (see Fig. 6B). Because the temporal integration window is short in SI [Mauguière et al., 1997] and long in SII [Wühle et al., 2011] any selective interaction for short delays is more compatible with interactions occurring within SI, rather than top-down modulations via higher level processing. In agreement with our hypothesis, this result is compatible with the notion that somatosensory inputs from opposite body sides can interact at early stages of tactile processing most likely through transcallosal pathways connecting SI in the two hemispheres (Fig. 1B).

\section{Integration of Tactile Stimuli From Same and Different Sides of the Body}

Similar to previous MEG studies, we found neuromagnetic activity in SI contralateral to the probe stimulus and 
in bilateral SII [Hari et al., 1993; Hoechstetter et al., 2001; Maldjian et al., 1999; Wühle et al., 2011], whereas no activity in the iSI was found, corroborating the notion of the contralateral vocation of SI and the bilateral nature of SII. When adaptor and probe stimulus were delivered on the same hand, unilateral stimulation response suppression was comparable in cSII and cSI. By contrast, when adaptor and probe were delivered on opposite hands, response suppression (i.e., our proxy of the interactions between adaptor and probe stimuli) was stronger in cSII compared with cSI. This result was largely expected and it likely reflects the existence of denser bilateral afferent projections in SII compared with SI [Forss et al., 1995; Hari et al., 1993; Lin and Forss, 2002]. The crucial aspect of our findings, however, is the observation that when adaptor and probe were on opposite hands, response suppression in cSI was nonetheless present.

Bilateral interactions in SI are in line with previous neurophysiological findings in monkeys [Iwamura et al., 2001; Killackey et al., 1983; Lipton et al., 2006] and with behavioral [Braun et al., 2005; Craig, 1985; D'Amour and Harris, 2013; Gescheider et al., 1970; Harrar et al., 2013; Harris et al., 2001; Nguyen et al., 2014; Sathian and Zangaladze, 1997, 1998; Sherrick, 1964; Tamè et al., 2011] and neuroimaging studies in humans [Hlushchuk and Hari, 2006; Kakigi, 1986; Kakigi and Jones, 1985; Nihashi et al., 2005; Noachtar et al., 1997; Sutherland and Tang, 2006; Tamè et al., 2012]. Previous findings from our laboratory [Tamè et al., 2011, 2012, 2013] have recognised the ability of SI to integrate inputs from the two sides of the body. However, the low temporal resolution of fMRI did not allow us to determine the time course of the interactions between contralateral and ipsilateral tactile stimulation in SI and SII, thus limiting any definite conclusion about the pathway/ flow of sensory information leading to the activations observed in SI in response to bilateral stimulation. In the present work, the high-temporal resolution of MEG combined with the RS paradigm sensitivity revealed that suppression in cSI in response to bilateral stimulation was weak when adaptor and probe were delivered simultaneously, emerged very clearly during the short-delay condition, and vanished at the long delay. This temporal profile of the response pattern in CSI under bilateral stimulation reveals that, differently from previous reports [Chung et al., 2014; Jung et al., 2012], responses to bilateral touch cannot be entirely ascribed to higher stages of processing, such as SII, because the suppression occurs very early in time [i.e., short delay (25ms)]. In other words, very fast suppression effects rule out the possibility that the activity we registered in cSI is a mere reflection of top-down modulation of cSI by well-known bilaterally organized higherlevel brain areas. Instead, our data suggest that it is more likely that tactile information from the stimulated body side reaches iSI via transcallosal connections already at the level of SI [Manzoni et al., 1989; Shuler et al., 2001; Tomasch, 1954]. In this respect, a recent tractography study has shown the presence of transcallosal fibre tracts connecting homologous sensorimotor cortical regions [Fling et al., 2013]. In addition, earlier bilateral interactions at subcortical and/or spinal cord level can be present, as shown by reports on patients that underwent callosotomy [Corballis, 1994; Sergent, 1990]. A recent electroencephalography (EEG) study carried out by Ragert and coworkers (2011) suggested the existence of interactions between ipsilateral and cSI after median nerve (MN) stimulation in an interval ranging between 20 and $25 \mathrm{~ms}$ post stimulus [Ragert et al., 2011]. Moreover, in a study on healthy individuals Korvenoja and coworkers (1995) reported iSI activation, in five of the ten participants, after MN stimulation in a window ranging between 80 and $300 \mathrm{~ms}$. The ipsilateral response was weaker compared with the contralateral one [Korvenoja et al., 1995]. Similarly, Zhu and coworkers (2007) studying the spatiotemporal integration of tactile information, using high-resolution MEG in a digit oddball paradigm, report early ipsilateral responses (i.e., $10 \mathrm{~ms}$ later than the contralateral response) in the anterior parietal field (Zhu et al., 2007). However, in these previous reports on healthy subjects early ipsilateral activity was not consistently present in all participants. Moreover, Kanno and coworkers (2004) in a study on two patients with a severe left brain damage reported ECDs of ipsilateral responses on the central sulcus after right MN stimulation. The dipole locations for right $\mathrm{MN}$ stimulation were adjacent to the location of the $\mathrm{N} 20 \mathrm{~m}$ response to left MN stimulation [Kanno et al., 2004]. Recently, Nevalainen and coworkers (2012), studying adolescents with cerebral palsy with MEG, reported the presence of ipsilateral responses in SI more often than in the control groups under MN stimulation even though with longer latency compared with the cSI [Nevalainen et al., 2012]. Finally, a single case study on a brain-damaged patient provided indications for integration of proprioceptive information from the two sides of the body also in the absence of SI in one of the two hemispheres [Borchers et al., 2011]. Although these findings on patients are insightful, individuals with a brain damage typically undergo some brain reorganization processes that could account, at least in part, for atypical effects. Our data instead reveal interactions between stimuli delivered to the two body sides detected as early as in SI of neurologically healthy controls.

The lack of any modulation in the neuromagnetic activity when bilateral stimulation was delivered with a longer delay (i.e., $125 \mathrm{~ms}$ ) might be the consequence of the fact that the window for bilateral integration in SI is shorter than the adopted delay. The maximal estimated permanence of the tactile signal in SI tends to be around $110 \mathrm{~ms}$ [Hamada et al., 2002], which is shorter than the $125 \mathrm{~ms}$ long-delay we adopted for comparative purposes. Therefore, our results provide further evidence, in addition to the one just described above in healthy and pathological individuals, in favour of an early integration of bilateral stimuli in SI under certain temporal constraints.

A further intriguing aspect of cSI response to bilateral stimulation is the modulation of response suppression as a 
function of adaptor finger. In particular, RS in cSI in response to bilateral stimulation was larger when homologous compared with non-homologous fingers were stimulated. This result extends the results of our previous fMRI study in which we have shown that repeated tactile stimulation of the same finger and homologous fingers of opposite hands produced an adaptation effect in the SI [Tamè et al., 2012]. Similarly, in a series of behavioural studies we have shown that masking effects vanish when homologous fingers of opposite hands are stimulated [Tamè et al., 2011, 2013]. This finger-specific pattern implies that not only SI can respond to bilateral stimulation, but also that it does so in a somatotopically organised manner-which constitutes further evidence that the integration of bilateral touch is occurring at the level of SI or earlier.

\section{Implications for the Neural Mechanisms Underlying Tactile Repetition Suppression}

One unexpected finding of the present work emerged in the profile of response suppression of cSI, when the adaptor and probe were delivered to the same hand. While non-homologous fingers stimulation produced smaller RS effect at the long compared with the short delay, homologous fingers stimulation produced comparable amount of suppression regardless of the delay (compare the two red bars in Fig. 6A). If RS response reflects a single underlying neural mechanism, we should have observed identical modulations as a function of delay for both homologous and non-homologous finger stimulations. Because this was clearly not the case, the hypothesis of a single mechanism mediating RS becomes unlikely. Instead, our data suggest that different neural mechanisms can mediate RS when tactile stimulation repeats exactly on the same finger (homologous condition), compared with when tactile stimulation occurs on neighbouring fingers (non-homologous condition). Below we discuss the possibility that RS effects for homologous fingers reflect neural adaptation, whereas RS effects for non-homologous fingers reflect lateral inhibition.

Previous works that measured RS to repeated tactile stimulation on the same body part have linked this response to the phenomenon of neural adaptation (Otsuru et al., 2011; Tamè et al., 2012; Tanaka et al., 1991). This interpretation is in agreement with previous interpretations of RS in other sensory modalities [Grill-Spector and Malach, 2001; Otsuru et al., 2011; Tamè et al., 2012; Tanaka et al., 1991]. Of particular interest are two previous MEG studies that examined reduction in the neuromagnetic activity following repeated stimulation of the same finger, when different delays between stimuli were applied [Wühle et al., 2010, 2011]. Wühle and coworkers (2011) found that when a tactile stimulus (i.e., probe) was preceded by a near- or supra-threshold stimulus (i.e., adaptor) on the same finger, the dipole activity evoked by the probe stimulus was reduced compared with when the probe stimulus was presented alone. More interestingly, the adaptation effect was the same regardless of whether the delay between the stimuli was $80 \mathrm{~ms}$ or $100 \mathrm{~ms}$ (for similar results see also Wühle et al., 2010). Similarly, in the present work in the unilateral stimulation condition the response suppression was the same with comparatively shorter and longer temporal delays (i.e., $25 \mathrm{~ms}$ and 125 $\mathrm{ms}$ ). These findings suggest that in SI the somatosensory processing of repeated tactile stimulations of the same finger starts to be integrated as early as $25 \mathrm{~ms}$ and this integration can occur at least up to $125 \mathrm{~ms}$. Instead, under bilateral stimulation the RS was no longer present at the latter delay. Therefore, the adaptation effect indicates that the persistence of tactile signals in SI coming from the same region of the skin is lasting longer (i.e., at least 125 $\mathrm{ms})$, as compared with when tactile stimuli are delivered to a different region, even if the latter concerns the homologous finger, but of opposite hands (i.e., at least $25 \mathrm{~ms}$ ).

The suppression effect for repeated touches delivered to different fingers has been studied to a much lesser extent. To our knowledge, our previous fMRI report was the only study that examined RS in non-homologous conditions [Tamè et al., 2012]. The effect appears to be broadly consistent with a lateral inhibition phenomenon [Biermann et al., 1998; Cardini et al., 2011], first described by von Bekesy (1967) in his seminal book "Sensory Inhibition", precisely for the tactile modality [Von Békésy, 1967; Moore and Nelson, 1998; Zhu and Connors, 1999]. Lateral inhibition is a neurophysiological effect determined by the firing of neurons responding to a certain region with a consequent reduction of the neuronal activity in the neighbouring regions [DiCarlo et al., 1998]. For instance, when a region of the skin is stimulated (e.g., index finger), the neuronal population responding to that region is activated and its response inhibits the activity from neighboring neurons such as, for instance, those responding to the middle finger [Geldard and Sherrick, 1972] (for a discussion on sensory inhibition mechanisms, see [Von Békésy, 1967]. Other studies have shown that suppressive response in the somatosensory cortex under double tactile stimulation depends on lateral inhibitory processing both within [Laskin and Spencer, 1979] and between [Okajima et al., 1991] the hands. Although we are not aware of studies that examined the possible modulation of lateral inhibition processes as a function of time, von Bekesy (1967) reported an observation that suggests that lateral inhibition is modulated as a function of the delay between the stimuli. "If two vibrators are placed $12 \mathrm{~cm}$ apart on the arm, and are actuated with a series of clicks without time difference, the vibration will be localized in the middle of the two vibrators as a rather diffuse sensation. When a time difference between the vibrators is introduced, the sensation will move toward the vibrator that receives the click earlier. At the same time the lateral spreading of the vibratory sensation becomes smaller" [Von Békésy, 1967].

Similarly, our data show that tactile stimulation of neighboring fingers when a delay is introduced, reduce 
the suppression to the delay between the tactile stimuli. In particular, the greater was the delays the lower was the suppression, and therefore lateral inhibition. This inhibitory effect as a function of delay was occurring at the level of SI (Fig. 6A), but not at the level of SII (Fig. 7A,C) where the inhibition was the same for the different delays. This is consistent with suppression studies of somatosensory cortex excitability which show that in SI the longer the delay between two afferent stimuli the smaller the reduction in the amplitude of the somatosensory evoked/field potential [McLaughlin and Kelly, 1993; Stevenson et al., 2012]. However, this pattern is known to be absent in SII [Wühle et al., 2011]. As to this pattern observed in SII, we do not have straightforward explanations. Nonetheless, we feel that it is important to report this secondary finding for completeness and to stimulate further studies on the interactions between SI and SII. It would be tempting to speculate that the pattern observed in SII reflects the propagation of the inhibitory effects of SI, modulated further by the less clear somatotopy of SII. For instance, any lateral inhibition effect observed in SI could become a form of RS in SII-due to the less clear distinction between fingers.

\section{CONCLUSIONS}

Our MEG measure of brain activity during a RS paradigm showed that tactile stimuli coming from the two sides of the body are integrated within the SI shortly after the stimuli came in contact with the skin. This observation moves our understanding of bilateral touch processing forward by showing that in humans, despite its primarily contralateral response, SI is capable of integrating touches from opposite body sides at early stages of somatosensory information processing. This early integration is compatible with bilateral touch reaching SI more likely through transcallosal inputs from cSI. The involvement of SI in the integration of bilateral touch is also strengthened by the fact that RS was modulated by the relative position of the stimuli on the hands (homologous vs. non-homologous finger), both in unilateral and in bilateral stimulation. This finger specificity points to the involvement of a brain structure characterised by high degrees of somatotopy, such as SI. Interestingly, during unilateral stimulation, this modulation of RS as a function of stimulated fingers changed also as a function of time: persisting across short and long delays when the same finger was stimulated twice, but decaying at the long delay when neighbouring fingers were stimulated. This unexpected finding may indicate that distinct neural mechanisms operate under the RS measure. Neural adaptation processes may account for RS effects following repeated stimulation of the same skin region (homologous fingers); in addition, lateral inhibitory processes may concur, with a different temporal profile, to the overall RS effect when tactile stimulations are presented on different skin regions (non-homologous fingers). We speculate that such SI mechanisms, by providing complementary spatiotemporal information may be functional to an appropriate sensory feedback while coordinating manipulative actions.

\section{ACKNOWLEDGMENT}

The authors are grateful to Dr. Francesco Prantil for the conditions randomization program. They thank Drs. Gianpaolo Demarchi and Gianpiero Monittola for the technical support. They thank the editors, and two anonymous reviewers for their insightful comments on a previous version of the manuscript.

\section{REFERENCES}

Aboitiz F, Scheibel AB, Fisher RS, Zaidel E (1992): Fiber composition of the human corpus callosum. Brain Res 598:143-153.

Allison T, McCarthy G, Wood CC, Williamson PD, Spencer DD (1989): Human cortical potentials evoked by stimulation of the median nerve. II. Cytoarchitectonic areas generating longlatency activity. J Neurophysiol 62:711-722.

Belin P, Zatorre RJ (2003): Adaptation to speaker's voice in right anterior temporal lobe. Neuroreport 14:2105-2109.

Biermann K, Schmitz F, Witte OW, Konczak J, Freund HJ, Schnitzler A (1998): Interaction of finger representation in the human first somatosensory cortex: A neuromagnetic study. Neurosci Lett 251:13-16.

Borchers S, Hauser T-K, Himmelbach M (2011): Bilateral hand representations in human primary proprioceptive areas. Neuropsychologia 49:3383-3391.

Brainard DH (1997): The Psychophysics Toolbox. Spat Vis 10:433436.

Braun C, Hess H, Burkhardt M, Wühle A, Preissl H (2005): The right hand knows what the left hand is feeling. Exp Brain Res 162:366-373.

Caminiti R, Carducci F, Piervincenzi C, Battaglia-Mayer A, Confalone G, Visco-Comandini F, Pantano P, Innocenti GM (2013): Diameter, length, speed, and conduction delay of callosal axons in macaque monkeys and humans: Comparing data from histology and magnetic resonance imaging diffusion tractography. J Neurosci 33:14501-14511.

Cardini F, Longo MR, Haggard P (2011): Vision of the body modulates somatosensory intracortical inhibition. Cereb Cortex 21: 2014-2022.

Chong TT-J, Cunnington R, Williams MA, Kanwisher N, Mattingley JB (2008): fMRI adaptation reveals mirror neurons in human inferior parietal cortex. Curr Biol 18:1576-1580.

Chung YG, Han SW, Kim H-S, Chung S-C, Park J-Y, Wallraven C, Kim S-P (2014): Intra- and inter-hemispheric effective connectivity in the human somatosensory cortex during pressure stimulation. BMC Neurosci 15:43.

Corballis MC (1994): Split decisions: Problems in the interpretation of results from commissurotomized subjects. Behav Brain Res 64:163-172.

Craig JC (1985): Attending to two fingers: Two hands are better than one. Percept Psychophys 38:496-511.

D'Amour S, Harris LR (2013): Contralateral tactile masking between forearms. Exp Brain Res 2014;232:821-826. 
Dehaene-Lambertz G, Dehaene S, Anton J-L, Campagne A, Ciuciu P, Dehaene GP, Denghien I, Jobert A, Lebihan D, Sigman M, Pallier C, Poline J-B (2006): Functional segregation of cortical language areas by sentence repetition. Hum Brain Mapp 27:360-371.

DiCarlo JJ, Johnson KO, Hsiao SS (1998): Structure of receptive fields in area $3 \mathrm{~b}$ of primary somatosensory cortex in the alert monkey. J Neurosci 18:2626-2645.

Eickhoff SB, Jbabdi S, Caspers S, Laird AR, Fox PT, Zilles K, Behrens TEJ (2010): Anatomical and functional connectivity of cytoarchitectonic areas within the human parietal operculum. J Neurosci 30:6409-6421.

Fabri M, Polonara G, Del Pesce M, Quattrini A, Salvolini U, Manzoni T (2001): Posterior corpus callosum and interhemispheric transfer of somatosensory information: An fMRI and neuropsychological study of a partially callosotomized patient. J Cogn Neurosci 13:1071-1079.

Fabri M, Del Pesce M, Paggi A, Polonara G, Bartolini M, Salvolini U, Manzoni T (2005): Contribution of posterior corpus callosum to the interhemispheric transfer of tactile information. Brain Res Cogn Brain Res 24:73-80.

Farnè A, Brozzoli C, Làdavas E, Ro T (2007): Investigating multisensory spatial cognition through the phenomenon of extinction. In: Haggard, P, Rossetti, Y, Kawato, M, editors. Sensorimotor Foundations of Higher Cognition. Oxford University Press.

Fling BW, Benson BL, Seidler RD (2013): Transcallosal sensorimotor fiber tract structure-function relationships. Hum Brain Mapp 34:384-395.

Forss N, Jousmäki V, Hari R (1995): Interaction between afferent input from fingers in human somatosensory cortex. Brain Res 685:68-76.

Geldard FA, Sherrick CE (1972): The cutaneous "rabbit": A perceptual illusion. Science 178:178-179.

Gescheider GA, Herman DD, Phillips JN (1970): Criterion shifts in the measurement of tactile masking. Percept Psychophys 433436.

Grill-Spector K, Malach R (2001): fMR-adaptation: A tool for studying the functional properties of human cortical neurons. Acta Psychol (Amst) 107:293-321.

Grill-Spector K, Henson R, Martin A (2006): Repetition and the brain: Neural models of stimulus-specific effects. Trends Cogn Sci (Regul Ed) 10:14-23.

Gross CG, Rocha-Miranda CE, Bender DB (1972): Visual properties of neurons in inferotemporal cortex of the Macaque. J Neurophysiol 35:96-111.

Hamada Y, Otsuka S, Okamoto T, Suzuki R (2002): The profile of the recovery cycle in human primary and secondary somatosensory cortex: A magnetoencephalography study. Clin Neurophysiol 113:1787-1793.

Hari R, Reinikainen K, Kaukoranta E, Hämäläinen M, Ilmoniemi R, Penttinen A, Salminen J, Teszner D (1984): Somatosensory evoked cerebral magnetic fields from SI and SII in man. Electroencephalogr Clin Neurophysiol 57:254-263.

Hari R, Karhu J, Hämäläinen M, Knuutila J, Salonen O, Sams M, Vilkman V (1993): Functional organization of the human first and second somatosensory cortices: A neuromagnetic study. Eur J Neurosci 5:724-734.

Harrar V, Spence C, Makin TR (2013): Topographic generalization of tactile perceptual learning. J Exp Psychol Hum Percept Perform 40:15-23.

Harris JA, Harris IM, Diamond ME (2001): The topography of tactile working memory. J Neurosci 21:8262-8269.
Henson RNA (2003): Neuroimaging studies of priming. Prog Neurobiol 70:53-81.

Hlushchuk Y, Hari R (2006): Transient suppression of ipsilateral primary somatosensory cortex during tactile finger stimulation. J Neurosci 26:5819-5824.

Hoechstetter K, Rupp A, Stancák A, Meinck HM, Stippich C, Berg $P$, Scherg M (2001): Interaction of tactile input in the human primary and secondary somatosensory cortex-a magnetoencephalographic study. Neuroimage 14:759-767.

Iwamura Y, Taoka M, Iriki A (2001): Bilateral activity and callosal connections in the somatosensory cortex. Neuroscientist 7:419429.

Iwamura Y, Tanaka M, Iriki A, Taoka M, Toda T (2002): Processing of tactile and kinesthetic signals from bilateral sides of the body in the postcentral gyrus of awake monkeys. Behav Brain Res 135:185-190.

Jacobs S, Brozzoli C, Hadj-Bouziane F, Meunier M, Farnè A (2011): Studying multisensory processing and its role in the representation of space through pathological and physiological crossmodal extinction. Front Psychol 2:89.

Jung P, Klein JC, Wibral M, Hoechstetter K, Bliem B, Lu M-K, Wahl M, Ziemann U (2012): Spatiotemporal dynamics of bimanual integration in human somatosensory cortex and their relevance to bimanual object manipulation. J Neurosci 32:56675677.

Kakigi R (1986): Ipsilateral and contralateral SEP components following median nerve stimulation: Effects of interfering stimuli applied to the contralateral hand. Electroencephalogr Clin Neurophysiol 64:246-259.

Kakigi R, Jones SJ (1985): Effects on median nerve SEPs of tactile stimulation applied to adjacent and remote areas of the body surface. Electroencephalogr Clin Neurophysiol 62:252-265.

Kanno A, Nakasato N, Hatanaka K, Yoshimoto T (2003): Ipsilateral area $3 \mathrm{~b}$ responses to median nerve somatosensory stimulation. Neuroimage 18:169-177.

Kanno A, Nakasato N, Nagamine Y, Tominaga T (2004): Nontranscallosal ipsilateral area $3 \mathrm{~b}$ responses to median nerve stimulus. J Clin Neurosci 11:868-871.

Killackey HP, Gould HJ III, Cusick CG, Pons TP, Kaas JH (1983): The relation of corpus callosum connections to architectonic fields and body surface maps in sensorimotor cortex of new and old world monkeys. J Comp Neurol 219:384-419.

Korvenoja A, Wikstrom H, Huttunen J, Virtanan J, Laine P, Aronen HJ, Seppalainen AM, Ilmoniemi RJ (1995): Activation of ipsilateral primary sensorimotor cortex by median nerve stimulation. Neuroreport 6:2589-2593.

Kourtzi Z, Kanwisher N (2000): Cortical regions involved in perceiving object shape. J Neurosci 20:3310-3318.

Krekelberg B, Boynton GM, van Wezel RJA (2006): Adaptation: From single cells to BOLD signals. Trends Neurosci 29:250256.

Laskin SE, Spencer WA (1979): Cutaneous masking. I. Psychophysical observations on interactions of multipoint stimuli in man. J Neurophysiol 42:1048-1060.

Li L, Miller EK, Desimone R (1993): The representation of stimulus familiarity in anterior inferior temporal cortex. J Neurophysiol 69:1918-1929.

Li Hegner Y, Lee Y, Grodd W, Braun C (2010): Comparing tactile pattern and vibrotactile frequency discrimination: A human FMRI study. J Neurophysiol 103:3115-3122. 
Lin YY, Forss N (2002): Functional characterization of human second somatosensory cortex by magnetoencephalography. Behav Brain Res 135:141-145.

Lingnau A, Ashida H, Wall MB, Smith AT (2009a): Speed encoding in human visual cortex revealed by fMRI adaptation. J Vis 9:3.1-14.

Lingnau A, Gesierich B, Caramazza A (2009b): Asymmetric fMRI adaptation reveals no evidence for mirror neurons in humans. Proc Natl Acad Sci USA 106:9925-9930.

Lipton ML, Fu K-MG, Branch CA, Schroeder CE (2006): Ipsilateral hand input to area $3 \mathrm{~b}$ revealed by converging hemodynamic and electrophysiological analyses in macaque monkeys. J Neurosci 26:180-185.

Mahon BZ, Milleville SC, Negri GAL, Rumiati RI, Caramazza A, Martin A (2007): Action-related properties shape object representations in the ventral stream. Neuron 55:507-520.

Maldjian JA, Gottschalk A, Patel RS, Detre JA, Alsop DC (1999): The sensory somatotopic map of the human hand demonstrated at 4 Tesla. Neuroimage 10:55-62.

Manzoni T, Barbaresi P, Conti F, Fabri M (1989): The callosal connections of the primary somatosensory cortex and the neural bases of midline fusion. Exp Brain Res 76:251-266.

Mauguière F, Merlet I, Forss N, Vanni S, Jousmäki V, Adeleine P, Hari R (1997): Activation of a distributed somatosensory cortical network in the human brain: A dipole modelling study of magnetic fields evoked by median nerve stimulation. Part II: Effects of stimulus rate, attention and stimulus detection. Electroencephalogr Clin Neurophysiol 104:290-295.

McLaughlin DF, Kelly EF (1993): Evoked potentials as indices of adaptation in the somatosensory system in humans: A review and prospectus. Brain Res Brain Res Rev 18:151-206.

Miller EK, Li L, Desimone R (1991): A neural mechanism for working and recognition memory in inferior temporal cortex. Science 254:1377-1379.

Moore CI, Nelson SB (1998): Spatio-temporal subthreshold receptive fields in the vibrissa representation of rat primary somatosensory cortex. J Neurophysiol 80:2882-2892.

Nelson AJ, Chen R (2008): Digit somatotopy within cortical areas of the postcentral gyrus in humans. Cereb Cortex 18:2341-2351.

Nevalainen P, Pihko E, Mäenpää H, Valanne L, Nummenmaa L, Lauronen L (2012): Bilateral alterations in somatosensory cortical processing in hemiplegic cerebral palsy. Dev Med Child Neurol 54:361-367.

Nguyen RH, Forshey TM, Holden JK, Francisco EM, Kirsch B, Favorov O, Tommerdahl M (2014): Vibrotactile discriminative capacity is impacted in a digit-specific manner with concurrent unattended hand stimulation. Exp Brain Res.

Nihashi T, Naganawa S, Sato C, Kawai H, Nakamura T, Fukatsu H, Ishigaki T, Aoki I (2005): Contralateral and ipsilateral responses in primary somatosensory cortex following electrical median nerve stimulation-An fMRI study. Clin Neurophysiol 116:842-848.

Noachtar S, Lüders HO, Dinner DS, Klem G (1997): Ipsilateral median somatosensory evoked potentials recorded from human somatosensory cortex. Electroencephalogr Clin Neurophysiol 104:189-198.

Okajima Y, Chino N, Saitoh E, Kimura A (1991): Interactions of somatosensory evoked potentials: simultaneous stimulation of two nerves. Electroencephalogr Clin Neurophysiol 80:26-31.

Otsuru N, Inui K, Yamashiro K, Urakawa T, Keceli S, Kakigi R (2011): Effects of prior sustained tactile stimulation on the soma- tosensory response to the sudden change of intensity in humans: An magnetoencephalography study. Neuroscience 182:115-124.

Papadelis C, Eickhoff SB, Zilles K, Ioannides AA (2011): BA3b and BA1 activate in a serial fashion after median nerve stimulation: Direct evidence from combining source analysis of evoked fields and cytoarchitectonic probabilistic maps. Neuroimage 54:60-73.

Penfield W, Boldrey E (1937): Somatic motor and sensory representation in the cerebral cortex of man as studied by electrical stimulation. Brain 60:389-443.

Ragert P, Nierhaus T, Cohen LG, Villringer A (2011): Interhemispheric interactions between the human primary somatosensory cortices. PLoS ONE 6:e16150.

Reed JL, Qi H-X, Zhou Z, Bernard MR, Burish MJ, Bonds AB, Kaas JH (2010): Response properties of neurons in primary somatosensory cortex of owl monkeys reflect widespread spatiotemporal integration. J Neurophysiol 103:2139-2157.

Reed JL, Qi H-X, Kaas JH (2011): Spatiotemporal properties of neuron response suppression in owl monkey primary somatosensory cortex when stimuli are presented to both hands. J Neurosci 31:3589-3601.

Sathian K, Zangaladze A (1997): Tactile learning is task specific but transfers between fingers. Percept Psychophys 59: 119-128.

Sathian K, Zangaladze A (1998): Perceptual learning in tactile hyperacuity: Complete intermanual transfer but limited retention. Exp Brain Res 118:131-134.

Schnitzler A, Salmelin R, Salenius S, Jousmäki V, Hari R (1995): Tactile information from the human hand reaches the ipsilateral primary somatosensory cortex. Neurosci Lett 200:25-28.

Schwarzbach J (2011): A simple framework (ASF) for behavioral and neuroimaging experiments based on the psychophysics toolbox for MATLAB. Behav Res Methods. Available at: http:/ / www.ncbi.nlm.nih.gov/pubmed/21614662.

Sergent J (1990): Furtive incursions into bicameral minds. Integrative and coordinating role of subcortical structures. Brain 113 (Pt 2):537-568.

Sherrick CE Jr (1964): Effects of double simultaneous stimulation of the skin. Am J Psychol 77:42-53.

Shuler MG, Krupa DJ, Nicolelis MA (2001): Bilateral integration of whisker information in the primary somatosensory cortex of rats. J Neurosci 21:5251-5261.

Simons SB, Chiu J, Favorov OV, Whitsel BL, Tommerdahl M (2007): Duration-dependent response of SI to vibrotactile stimulation in squirrel monkey. J Neurophysiol 97:2121-2129.

Stancak A, Hoechstetter K, Tintera J, Vrana J, Rachmanova R, Kralik J, Scherg M (2002): Source activity in the human secondary somatosensory cortex depends on the size of corpus callosum. Brain Res 936:47-57.

Stevenson CM, Wang F, Brookes MJ, Zumer JM, Francis ST, Morris PG (2012): Paired pulse depression in the somatosensory cortex: Associations between MEG and BOLD fMRI. Neuroimage 59:2722-2732.

Sutherland MT (2006): The hand and the ipsilateral primary somatosensory cortex. J Neurosci 26:8217-8218.

Sutherland MT, Tang AC (2006): Reliable detection of bilateral activation in human primary somatosensory cortex by unilateral median nerve stimulation. Neuroimage 33:1042-1054.

Tamè L, Farnè A, Pavani F (2011): Spatial coding of touch at the fingers: Insights from double simultaneous stimulation within and between hands. Neurosci Lett 487:78-82. 
Tamè L, Braun C, Lingnau A, Schwarzbach J, Demarchi G, Li Hegner Y, Farnè A, Pavani F (2012): The contribution of primary and secondary somatosensory cortices to the representation of body parts and body sides: An fMRI adaptation study. J Cogn Neurosci 24:2306-2320.

Tamè L, Farnè A, Pavani F (2013): Vision of the body and the differentiation of perceived body side in touch. Cortex 49:13401351.

Tanaka K, Saito H, Fukada Y, Moriya M (1991): Coding visual images of objects in the inferotemporal cortex of the macaque monkey. J Neurophysiol 66:170-189.

Tan H-RM, Wühle A, Braun C (2004): Unilaterally applied stimuli in a frequency discrimination task are represented bilaterally in primary somatosensory cortex. Neurol Clin Neurophysiol 2004:83.

Tian X, Poeppel D (2013): The effect of imagination on stimulation: The functional specificity of efference copies in speech processing. J Cogn Neurosci 25:1020-1036.

Tomasch J (1954): Size, distribution, and number of fibres in the human corpus callosum. Anat Rec 119:119-135.
Tommerdahl M, Simons SB, Chiu JS, Favorov O, Whitsel BL (2006): Ipsilateral input modifies the primary somatosensory cortex response to contralateral skin flutter. J Neurosci 26: 5970-5977.

Von Békésy G (1967): Sensory Inhibition. Princeton University Press.

Vuilleumier P, Henson RN, Driver J, Dolan RJ (2002): Multiple levels of visual object constancy revealed by event-related fMRI of repetition priming. Nat Neurosci 5:491-499.

Wühle A, Mertiens L, Rüter J, Ostwald D, Braun C (2010): Cortical processing of near-threshold tactile stimuli: An MEG study. Psychophysiology 47:523-534.

Wühle A, Preissl H, Braun C (2011): Cortical processing of nearthreshold tactile stimuli in a paired-stimulus paradigm-An MEG study. Eur J Neurosci 34:641-651.

Zhu J, Connors BW (1999): Intrinsic firing patterns and whiskerevoked synaptic responses of neurons in the rat barrel cortex. J Neurophysiol 81:1171-1183.

Zhu Z, Disbrow EA, Zumer JM, McGonigle DJ, Nagarajan SS (2007): Spatiotemporal integration of tactile information in human somatosensory cortex. BMC Neurosci 8:21. 